Revista Docência do Ensino

Superior

v. 2, 2012

\section{EL USO DE LOS PORTAFOLIOS EN EL APRENDIZAJE DE LA DISCIPLINA DE ADMINISTRACIÓN COMO ESTRATEGIA INNOVADORA EN EL AULA}

\section{RESUMEN}

Este trabajo presenta el impacto del uso de los portafolios en el aprendizaje de la disciplina de administración durante la formación del pregrado de los ingenieros de sistemas e informática de una universidad de Lima Norte. El artículo corresponde a una investigación de diseño cuasi experimental en la que se implementó el Portafolio del estudiante, de tipo semi estructurado a un Grupo Experimental conformado por 18 estudiantes. Para determinar el impacto del Portafolio del estudiante en el aprendizaje de la disciplina de administración, se utilizó un instrumento validado denominado "Prueba objetiva", aplicado al Grupo Control como al Grupo Experimental en dos momentos: pre-test y pos-test. Para evaluar el uso de los Portafolios empleados por los estudiantes se utilizo la Rúbrica. Para el análisis cuantitativo de los datos se aplicaron pruebas no paramétricas (U de Mann Whitney), cuyos resultados obtenidos tanto en el pre test como en el post test, se evidenciaron cambios significativos en los niveles de aprendizaje del Grupo Experimental frente al Grupo Control. Cualitativamente también se destaca el impacto del Portafolio del estudiante en el Grupo Experimental, resaltando que la planificación, organización, dirección y el control de las evidencias permitió que los estudiantes logren buenos niveles de aprendizaje de contenidos conceptuales, procedimentales y actitudinales en la disciplina de estudio, reflejándose en el aula un ambiente que propicia mejores formas de relacionamiento entre los estudiantes y con el profesor.

Palavras-Chave: Portafolio. Estrategias innovadoras en el aula. Evaluación formativa. Aprendizaje activo. 


\section{INTRODUCCIÓN}

Este artículo forma parte de una tesis de maestría, cuyo objeto de estudio fue determinar el impacto de los efectos del uso del portafolio del estudiante como estrategia innovadora en el aula que permita superar los diferentes problemas que afectan el aprendizaje de los estudiantes del cuarto semestre de la carrera profesional de ingeniería de sistemas e informática en una Universidad de Lima-Norte. En ese sentido la interrogante principal de la investigación es conocer: ¿de qué manera el uso del portafolio del estudiante mejora el aprendizaje la disciplina de administración en la formación de los ingenieros de sistemas e informática?

Durante dieciséis semanas se acompaño a los 18 estudiantes que conformaron el Grupo Experimental, los cuales fueron capacitados mediante un módulo, en el cual aprendieron a emplear los portafolios como metodología de acompañamiento en sus procesos de aprendizaje, hallándose como resultados finales al concluir el estudio, la adquisición de habilidades y capacidades para planear y organizar, así como una mayor responsabilidad y autonomía sobre su propio aprendizaje.

El trabajo se organiza en dos partes. En la primera parte se presenta la metodología de investigación, por medio de la cual identifican las diversas dificultades y carencias que se presentan en los estudiantes al iniciar sus estudios de la disciplina, destacándose la falta de autonomía, las dificultades para trabajar y relacionarse con otros y la ausencia de la reflexión crítica sobre lo que aprenden.

En la segunda parte abordaremos acerca del uso de los portafolios en el ámbito educativo y los principales impactos producidos en los estudiantes que lo han incorporado en su actividad diaria para el estudio y aprendizaje de los contenidos, así como en la nueva configuración de su identidad frente al estudio y el aprendizaje autónomo.

\section{EL PORTAFOLIO EN LA DISCIPLINA DE ADMINISTRACIÓN}

La disciplina de administración es una de las seis disciplinas comprendidas en la malla curricular del cuarto ciclo de la carrera de ingeniería e informática. De naturaleza teórica y práctica, comprende cuatro unidades de estudio, desarrollados a través de sesiones de clase y trabajo independiente además del uso de los portafolios como metodo- 
logía de acompañamiento de aprendizaje. La disciplina tiene como objetivo general: conocer, explicar y aplicar los conceptos, técnicas y herramientas de la administración en la solución de problemas organizacionales.

El empleo de los portafolios como metodología de acompañamiento de aprendizajes a través del "módulo del portafolio", está dividido en cuatro dimensiones que la conforman: la planificación de las evidencias, la organización de las evidencias, la dirección de evidencias y el control de las evidencias. Mediante el cual se pretende que los estudiantes apliquen el proceso administrativo en su construcción y adquieran contenidos conceptuales, procedimentales y actitudinales relacionados con la administración. Fomentando el trabajo autónomo, el trabajo colaborativo y la socialización de sus logros, posibilitando una mayor participación del estudiante en su aprendizaje y un rol mediador del profesor en el proceso educativo.

El tipo de investigación realizada es cuasi experimental, para ello se conformo dos grupos de estudio homogéneos en edad (19 años promedio), sexo (14 varones y 4 damas) y en cantidad (18 estudiantes), denominados: Grupo Control y Grupo Experimental, este último, son a quienes se les aplicó el "módulo del portafolio". Los criterios de inclusión establecen que ambos grupos de estudiantes estén matriculados en el cuarto semestre de la carrera profesional de ingeniería de sistemas e informática y llevar la disciplina.

A fin de encontrar problemática en sus aprendizajes, nos entrevistamos con diferentes actores del proceso educativo. La tutora responsable de estos grupos fue el primer entrevistado, por tener una cercanía permanente con los grupos desde el primer ciclo de estudio. En la entrevista ella menciono que los estudiantes afrontan dificultades en el aprendizaje de contenidos educativos y específicos. Denotan aprendizajes mecánicos y repetitivos. Abordan los temas de estudio sin profundizar en su comprensión. Carecen de metodologías que acompañen sus procesos de aprendizaje, organización y estructura de sus ideas. Muy pocos son los que hacen uso de estrategias de aprendizajes para elaborar síntesis, resúmenes e informes.

También se consultaron otras opiniones, como la de otros profesores de disciplinas del mismo semestre y carrera, quienes manifestaron que los estudiantes manifiestan limitada responsabilidad y autonomía sobre su propio aprendizaje, presentando dificultades para socializar sus trabajos, elaborar informes, seleccionar, organizar materiales y sólo algunos tímidamente reflexionan sobre lo que aprenden, además de la 
ausencia de habilidades para planificar y organizar su tiempo para dar cuenta de las actividades requeridas y cambiar su dinámica de trabajo cuando estas no funcionan.

Los otros entrevistados fueron los propios estudiantes, los cuales reconocieron que tienen dificultades para llevar a la práctica lo que aprenden. Les resulta difícil aplicar la teoría a situaciones concretas. Algunos expresaron que si bien se plantean objetivos y metas, ellos no están seguros que las acciones que llevan a cabo les permitan alcanzarlos. Reconocen sus limitaciones para organizar, clasificar y seleccionar adecuadamente la información. A menudo el tiempo no les alcanza para cumplir con sus tareas y mucho menos para reflexionar. Para ellos trabajar en grupo es muy dificultoso, discuten y no dialogan, no consideran que pueden aprender de otros colegas, además de tener prejuicios para preguntar, exponer sus ideas y poco acercamiento al profesor. Señalan que es tarea de él evaluarlos y motivarlos para aprender.

Todos estos diálogos, fueron contrastados a lo largo del proceso de enseñanza aprendizaje, iniciándose con la realización del pre-test, aplicándose una prueba objetiva, cuyos resultados sobre 20 puntos, arrojaron puntajes de 5,8 para el Grupo Control y 6.3 en el Grupo Experimental. Además se aplicaron cuestionarios semi estructurados para recoger opiniones de los estudiantes. Todos los resultados al ser analizados, permitieron concluir que ambos grupos inicialmente tienen aprendizajes similares al iniciar el estudio de la disciplina.

Asimismo, al Grupo Experimental, quienes trabajaban con los portafolios, en la octava semana se les aplicó una Rúbrica a fin de evaluar el nivel de logros de aprendizajes procedimentales y actitudinales que venían alanzando. Los resultados obtenidos evidenciaron que aún persistían las dificultades para planear, organizarse y asumir con mayor autonomía su propio aprendizaje.

Por otro lado la búsqueda bibliográfica permitió encontrar respaldo teórico acerca de la situación presentada. Resaltándose los aportes de Altamirano Cárdenas (2006) y Benito Águeda (2005), quienes al referirse sobre las dificultades que se presentan en los estudiantes universitarios al aprender, ambos coinciden en destacar que ellos poseen una baja motivación para estudiar por ellos mismos, limitaciones para decidir sobre sus aprendizajes y están acostumbrados a la docencia magistral.

A fin de superar esta situación, Benito Águeda propone el empleo de metodologías activas, que les permitan a los profesores emplear adecuadamente el uso de los espacios de aprendizaje y recursos didácticos innovadores que fomenten en los estudi- 
antes un mayor interés por asumir su propio aprendizaje y reconocimiento de sus logros.

A través de estas prácticas se busca estimular la socialización de los trabajos y avances que logran los estudiantes, permitiendo que el profesor pueda brindar una mejor orientación y mediación, que conlleve a que el estudiante realice una mejor planificación de su tiempo y organización de recursos, evalué su propio desempeño y pueda cambiar de dinámica de trabajo cuando ésta no funciona.

Es en ese marco, que se plantea para el desarrollo de estas habilidades y destrezas a través del uso portafolio del estudiante, como una estrategia innovadora, un recurso didáctico, que contribuya a generar en ellos una mayor autonomía y la adquisición de nuevas capacidades para aprender.

\subsection{El uso de los portafolios en el ámbito educativo}

Al empezar el estudio acerca del uso del portafolio, nos remontamos a la década de los años 70, durante el proceso de la reforma educacional en los Estados Unidos, cuyos cambios apuntaron establecer que la evaluación en la escolaridad debía ser obligatoria y continua, personalizada e integrada al proceso educativo. Es así que las nuevas concepciones que se llevaron a cabo en la década de los 90, destacadas no sólo por lograr resultados cuantitativos, se pone un interés muy particular en lo cualitativo, en particular el conocer y evaluar el proceso de aprendizaje, por ende los conocimientos que van adquiriendo los alumnos. Es así que en esta década en ese país más de 500 instituciones de educación superior comienzan a utilizar los portafolios de aprendizaje como instrumento de evaluación (SELDIN, 2004).

García (2000), menciona que a pesar que el portafolio no surge en los ámbitos educativos, se han utilizado desde siempre. Hilda (1996) sostiene que la verdadera inclusión del portafolio en la educación se produce cuando aparece como una metodología alternativa frente aquellas de corte puramente cuantitativo, y se hace uso de ella como un proceso de evaluación de las trayectorias del aprendizaje, intentando entender más profundamente las habilidades y destrezas del estudiante mediante el conocimiento de las ejecuciones y logros obtenidos, incorporando además el valor añadido de reflexionar sobre su proceso y aumentar su potencial de aprendizaje.

A continuación destacamos tres investigaciones referentes sobre el uso de los portafolios en la enseñanza superior: 
Rezende (2010) destaca el efecto de los portafolios en el cambio de actitud de los estudiantes de una disciplina en una universidad pública de Brasil, evidenciando en los resultados, que su uso propició el desarrollo de la independencia del estudiante en relación con el profesor, lo volvió más responsable de su propio aprendizaje (dentro de una perspectiva de auto-aprendizaje y en una concepción socio interaccionista). Reflejándose en ellos una postura más investigativa, mayor percepción y consciencia sobre las consecuencias de sus errores, aproximándolos a generar una solución ideal de los errores y obstáculos que se le presentan, es decir, se manifestaron acciones que dieron cuenta que en él, se gestó un proceso de madures, en la forma de cómo afrontó el estudio y sus aprendizajes.

Villalustre y Del Moral Pérez (2010) profesores de la Universidad de Oviedo, quienes al aplicar metodologías activas y utilizar el portafolio como estrategia para el seguimiento del aprendizaje y evaluación, obtuvieron como resultados que el $80 \%$ de los estudiantes, potenciaron sus capacidades relacionadas con el recojo y organización de las evidencias; aumentaron sus destrezas para el análisis, la síntesis y el trabajo en equipo.

Pinar Sepúlveda y Morán (2006), quienes también demuestran con resultados positivos el empleo de esta herramienta en los 42 estudiantes de la Escuela Técnica Superior de Informática Aplicada en la Universidad Politécnica de Valencia, manifestaron que a través del portafolio, el profesor valoró sus esfuerzos sobre los procesos de recolección, clasificación y reflexión sobre sus aprendizajes. Comentando que obtuvieron una mayor concentración para el estudio y el aprendizaje de los conceptos además de una mejor aplicación de lo aprendido en la solución de problemas cotidianos.

La inclusión de los portafolios en la educación presenta varias posibilidades respecto a su uso; así encontramos aquellas que son referidas a su construcción por parte del discente, denominado "portafolio del estudiante", en donde el portafolio es una colección de sus producciones más representativas elegidas por él, además de reflexiones acerca de cómo están comprendiendo lo que estudian, permitiendo que el profesor pueda acompañarlo en su proceso de aprendizaje (VILLAS BOAS, 2001, p. 207). 


\subsection{Definiciones de los portafolios}

La Real Academia Española de la Lengua (RAE) define el portafolio como una "cartera de mano para llevar libros, papeles u otros materiales.". Aunque esta definición no está determinada en un contexto específico de la educación, consideramos señalarla.

Volviendo al contexto educativo, gestado de cambios y de innovaciones que durante los últimos años en muchas universidades se van produciendo, el portafolio esta usándose como instrumento de acompañamiento y mejoría de la enseñanza (REZENDE, 2010, p.30). En tal sentido el portafolio de aprendizaje es una herramienta pedagógica que permite la utilización de una metodología diferenciada y diversificada sobre el monitoreo y evaluación de los procesos de enseñanza y aprendizaje, sin dejar de lado la atención a la carga afectiva, inherente a dichas situaciones.

Siendo el portafolio un conjunto de acciones empleadas para el mejor resultado de la enseñanza y del aprendizaje (HERNÁNDEZ, 2000) sus resultados agregan valores al proceso por medio de la experiencia desarrollada dentro de un período de tiempo, por un análisis continuo durante la evolución de un proyecto, identificando posibles problemas que puedan ocurrir en su desarrollo.

Sá-Chaves (1998), quien al referirse al portafolio, lo define como un instrumento de construcción de conocimientos en el proceso de enseñanza aprendizaje, considerándose como una forma diagnóstica y continua de acompañamiento y evaluación de un determinado trabajo, en el que se pueden verificar y problematizar hipótesis en variadas situaciones.

Es así que los portafolios son colecciones de informaciones importantes que provienen de diversas fuentes: libros, revistas, periódicos, internet, testimonios de estudiantes, padres, profesores, funcionarios y demás personas envueltas en el trabajo. Fundamentados en registros (anotaciones) de la experiencia de los momentos de aprendizaje, dando relevancia y visibilidad al proceso formativo de adquisición y desarrollo de habilidades, capacidades, destrezas, actitudes y valores. Siendo su carácter comprensivo y de registro longitudinal que permite verificar dificultades y actuar en el proceso, en el tiempo del aprendizaje, de forma tal que se posibilite brindar ayuda la estudiante.

Para Klenowisk (2003) los portafolios posibilitan la comprensión tanto de la complejidad, como de las dinámicas inherentes al crecimiento del saber personal, valorizando y fomentando la reflexión sobre el aprendizaje, conduciendo a la metacogni- 
ción y la profundización del autoconocimiento. La utilización de los portafolios por los estudiantes universitarios a lo largo de un período de aprendizaje, pueden responder a un módulo, un ciclo o un semestre, permitiendo que los estudiantes, por sí mismos ejerciten una propuesta de evaluación más auténtica, participativa y reflexiva.

Otras definiciones acerca del portafolio citadas por Mellado (2005) lo identifican al portafolio como estrategia evaluativa y como una herramienta para atender los procesos de construcción del conocimiento. La diversidad de material presentado en un portafolio permite identificar diferentes aprendizajes, conceptos, procedimientos y actitudes, por tanto, proporciona una visión más amplia y más profunda de lo que los estudiantes saben y pueden hacer de sus competencias transversales y disciplinares (LYONS, 1999, GATICA, OREA Y VEGA, 2007).

Al culminar de citar las diferentes conceptualizaciones acerca de los portafolios, la definición del portafolio del estudiante corresponde a la creación de una carpeta de trabajo empleada por los estudiantes para recopilar y reflexionar sobre sus producciones o evidencias planificadas, organizadas, direccionadas y controladas hacia el logro de aprendizajes de contenidos conceptuales, procedimentales y actitudinales referidas a la asignatura de administración y organización.

Esta definición de portafolio establece que su producción es una creación auténtica porque en ella, el estudiante pone de manifiesto su identidad y el desarrollo de sus aprendizajes adquiridos (BARTON Y COLLINS, 1997), evidenciando su trabajo y esfuerzo por aprender, producto de una autoevaluación crítica y cuidadosa que envuelve los criterios de calidad de las producciones, las estrategias de aprendizaje que emplea, la comprensión individual y la interacción con otros compañeros, con el profesor, así como por la reflexión durante los momentos del trabajo individual y en grupo.

Entre los tipos y soportes que emplean los portafolios, Danielson y Abruntyn (2004) distinguen dos tipos de portafolio educativo: el portafolio profesional (de trabajo o de presentación) y el portafolio de enseñanza, los cuales son creados por profesores que trabajan con estudiantes.

En una segunda categoría de portafolio educativo, tenemos al portafolio del profesor y al portafolio del estudiante. Otras formas de tipificar a los portafolios son por su estructura: portafolio estructurado, en el que se establecen en forma anticipada la estructura final que tendrá la elaboración y presentación. El portafolio semi estructurado, el cual partiendo de una pauta inicial, permite que se incorporen nuevos elemen- 
tos, fomentando la creatividad y la participación del usuario al elaborar su carpeta reflexiva.

En lo que respecta a los soportes utilizados para presentar los portafolios, están: los portafolios impresos y el portafolio electrónico. Este último permite la incorporación de nuevos elementos audiovisuales, los cuales estarán regulados bajo criterios de ética y respeto de los derechos de autor a fin de evitar el copiado y pegado o la publicación de contenidos sin considerar las fuentes.

\subsection{Los portafolios en la disciplina de administración}

La implementación de los portafolios en la disciplina de administración se orienta a emplearse como una estrategia de acompañamiento para la enseñanza-aprendizaje de contenidos específicos de administración, promoviendo el desarrollo de las habilidades profesionales esenciales, como: la planificación y la organización, la ejecución y control, dentro del contexto cambiante del ambiente organizacional.

Su uso pretende que los estudiantes seleccionen, clasifiquen, ordenen e utilicen una serie de evidencias con la que confeccionan su carpeta reflexiva. Asimismo a través del trabajo con los portafolios, los estudiantes aprenderán a ser capaces de resolver problemas en la práctica, desarrollar habilidades analíticas, la reconstrucción imaginativa e independiente, el pensamiento reflexivo, necesarios en la actualidad para avanzar en el ámbito profesional (SHARMA, 1999).

En ese sentido, el portafolio del estudiante posee un alto potencial para desarrollar diversas habilidades y capacidades señaladas anteriormente y relacionadas también con las disciplinas de gestión.

\subsection{Metodología de aplicación de los portafolios}

El método de aplicación del portafolio en la disciplina de administración está dirigido al grupo de 18 estudiantes (Grupo Experimental), quienes participaron de un proceso de capacitación, mediante un módulo, a través del cual se les instruyó sobre su uso, pero también se les planteo espacios para el dialogo y la construcción conjunta, fomentando en ellos la iniciativa, el compromiso personal con su trabajo y una mayor responsabilidad en el desarrollo de sus propios hábitos de estudio y de aprendizaje. 
El objetivo buscado a través de esta metodología era lograr buenos niveles de aprendizaje en la disciplina, desarrollo de habilidades cognitivas y metacognitivas, frente a los otros estudiantes del Grupo control quienes no lo empleaban.

\section{RESULTADOS}

Al llegar la última semana de clases estimadas, se empezaron a realizar las evaluaciones respectivas, buscando conocer los logros alcanzados por ambos grupos, en especial al Grupo experimental, quienes emplearon los portafolios. Los hallazgos obtenidos en post test al aplicarse la prueba objetiva, para medir el nivel de logro de los aprendizajes conceptuales.

Cuadro 1 Promedios obtenidos al aplicarse la prueba objetiva en el pre test y post test

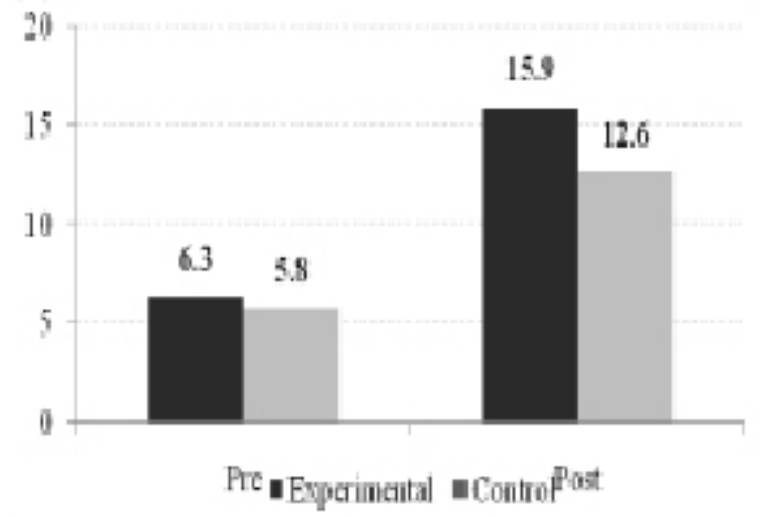

Los resultados evidencian mejoras significativas en los promedios finales entre ambos grupos. Destacándose el promedio de 15.9 obtenido por el Grupo experimental, que se traduce en un promedio de $27.2 \%$ mayor sobre el promedio de 12.5 que alcanzó el Grupo control (ver cuadro 1).

El nivel de logro alcanzado por el Grupo experimental corresponde a "buenos niveles de aprendizaje por encontrarse entre los valores de 14 a 16, según calificación de los niveles de aprendizaje conceptual (ver tabla 1). 
Tabla 1 - Clasificación de los niveles de aprendizaje conceptual a través de la prueba objetiva

\begin{tabular}{|c|c|c|c|c|}
\hline Aprendizaje Conceptual & $\begin{array}{l}\text { Instrumento } \\
\text { Evaluativo }\end{array}$ & & Niveles & $\begin{array}{l}\text { Calificación } \\
\text { (Vigésimal) }\end{array}$ \\
\hline \multirow{4}{*}{$\begin{array}{l}\text { Denominado también } \\
\text { conocimiento declarativo } \\
\text { o saber qué. } \\
\text { Para la presente } \\
\text { investigación corresponde } \\
\text { a la identificación y } \\
\text { reconocimiento correcto } \\
\text { de información referida a } \\
\text { los conceptos, técnicas y } \\
\text { herramientas de la } \\
\text { Administración }\end{array}$} & \multirow{4}{*}{ Prueba objetiva } & $\begin{array}{l}\text { Muy } \\
\text { Bueno }\end{array}$ & $\begin{array}{l}\text { Identifica correctamente } \\
\text { entre } 17 \text { a } 20 \text { ítems de la } \\
\text { Prueba objetiva. }\end{array}$ & De 17 a 20 \\
\hline & & Bueno & $\begin{array}{l}\text { Identifica correctamente } \\
\text { entre } 14 \text { a } 16 \text { ítems de la } \\
\text { Prueba objetiva. }\end{array}$ & De 14 a 16 \\
\hline & & Regular & $\begin{array}{l}\text { Identifica correctamente } \\
\text { entre } 11 \text { a } 13 \text { ítems de la } \\
\text { Prueba objetiva. }\end{array}$ & De 11 a 13 \\
\hline & & Deficiente & $\begin{array}{l}\text { Identifica correctamente } \\
\text { hasta } 10 \text { ítems de la } \\
\text { Prueba objetiva. }\end{array}$ & De 0 a 10 \\
\hline
\end{tabular}

Los impactos del portafolio del estudiante en los buenos niveles de aprendizajes alcanzado por el grupo experimental, se manifiestan en el aprendizaje procedimental. Resultados hallados al aplicarse la Rúbrica.

El cuadro 2 muestra los promedios que los estudiantes del grupo experimental alcanzaron en la presentación de sus portafolios. Destacándose que se producen mejoras porcentuales entre la primera y segunda presentación. Alcanzándose en la segunda presentación el 35.55 \% sobre los promedios de la primera presentación (ver cuadro 2).

Cuadro 2 - Promedios de los aprendizajes de contenidos procedimentales en la 1ra y 2da Presentación de los Portafolios

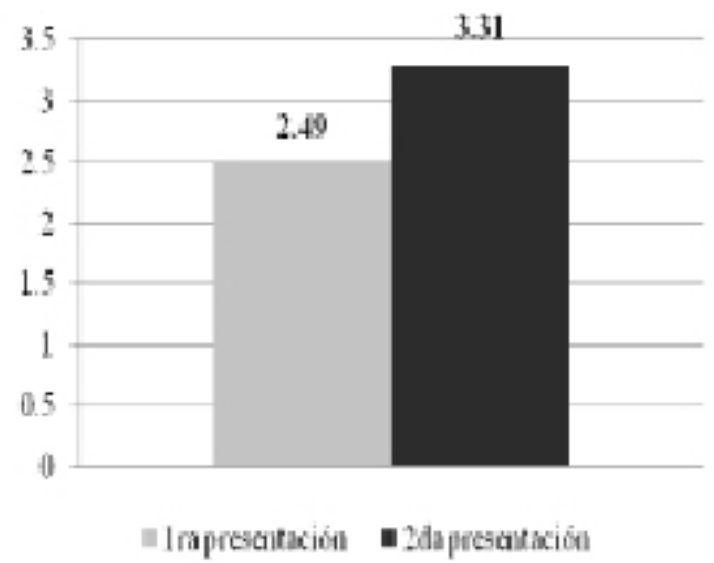


Los estudiantes en la primera presentación obtuvieron un promedio de 2.49 (ver cuadro 2), correspondiéndole la calificación de regular nivel de aprendizaje (según la tabla 2). Evidenciando que su aprendizaje procedimental aún estaba en desarrollo.

Tabla 2 - Clasificación de los niveles de aprendizaje procedimental a través de la Rúbrica

\begin{tabular}{|c|c|c|c|c|}
\hline $\begin{array}{l}\text { Aprendizaje } \\
\text { Conceptual }\end{array}$ & $\begin{array}{c}\text { Instrumento } \\
\text { Evaluativo }\end{array}$ & & Niveles & $\begin{array}{l}\text { Calificación } \\
\text { (Vigésimal) }\end{array}$ \\
\hline $\begin{array}{l}\text { Denominado también } \\
\text { saber hacer o saber } \\
\text { procedimental. } \\
\text { Para la presente }\end{array}$ & & Muy Bueno & $\begin{array}{l}\text { Elabora correctamente todos } \\
\text { los ítems de los indicadores } \\
\text { de cada dimensión del } \\
\text { Portafolio. }\end{array}$ & 4 \\
\hline $\begin{array}{l}\text { investigación } \\
\text { corresponde la } \\
\text { elaboración del listado } \\
\text { de actividades, }\end{array}$ & Rúbrica del & Bueno & $\begin{array}{l}\text { Elabora correctamente } \\
\text { algunos ítems de los } \\
\text { indicadores de cada } \\
\text { dimensión del Portafolio. }\end{array}$ & 3 \\
\hline $\begin{array}{l}\text { informes, mapas } \\
\text { conceptuales, gráficos, } \\
\text { entre otros, que dan } \\
\text { cuenta de la aplicación }\end{array}$ & Portafolio & Regular & $\begin{array}{l}\text { Elabora correctamente un } \\
\text { ítem de los indicadores de } \\
\text { cada dimensión del } \\
\text { Portafolio. }\end{array}$ & 2 \\
\hline $\begin{array}{l}\text { práctica de los } \\
\text { conceptos, técnicas y } \\
\text { herramientas de la } \\
\text { Administración }\end{array}$ & & Deficiente & $\begin{array}{l}\text { No elabora correctamente } \\
\text { ningún ítem de los } \\
\text { indicadores de cada } \\
\text { dimensión del Portafolio. }\end{array}$ & 1 \\
\hline
\end{tabular}

En el cuadro 3 se identifican los promedios obtenidos en cada indicador del aprendizaje procedimental entre ambas presentaciones. La primera presentación indica que las mayores dificultades se hallaron en los procesos y procedimientos relacionados a las dimensiones de Organización y Control (ambas con 2,44) y en la dimensión Dirección (2.22). 
Cuadro 3 - Promedios de los indicadores de aprendizaje de los contenidos procedimentales en la 1ra y 2da Presentación de los Portafolios

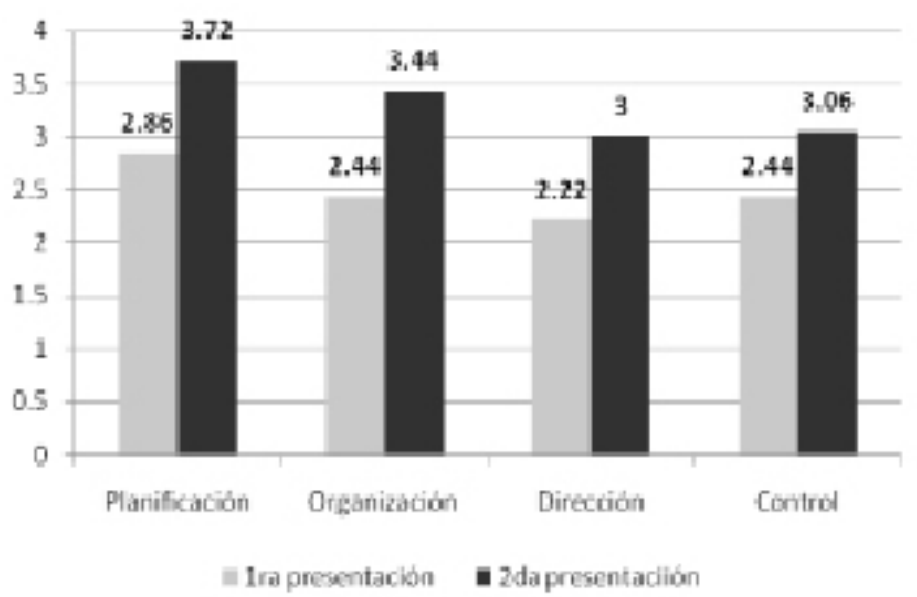

En la segunda presentación se destaca la mejoría de los promedios obtenidos en la dimensión Planificación, consolidándose con un porcentaje del 30\% sobre la primera, reflejándose en la capacidad de los estudiantes para establecer sus objetivos, plantear planes y elaborar cronogramas de actividades.

En las otras dimensiones también se producen mejoras. Los promedios en la dimensión Organización alcanzan el 40.98\% sobre la primera presentación, reflejándose en los estudiantes una mejor predisposición para organizar y clasificar correctamente sus evidencias, influyendo en el aprendizaje de los contenidos, esto a razón de que cuentan con producciones propias mejor clasificadas y ordenadas, facilitando el estudio, un mejor empleo del tiempo y los recursos.

La dimensión Dirección, la mejora de los promedios entre ambas presentaciones, destacan un incremento del 35\% sobre la primera, manifestándose en los estudiantes una mayor motivación para la reflexionar sobre sus logros, sus procesos de cómo van aprendiendo, el empleo de estrategias para aprender. Evidenciando la apropiación de aprendizajes referidos a los conceptos, técnicas y herramientas de la administración.

El trabajo cooperativo es considerado como un espacio para conocer nuevas opiniones y ampliar su conocimiento e interactuar con otros a través del dialogo, generándoles también una mayor confianza y afianzamiento de sus vínculos como grupo al exponer sus logros individuales al grupo. Información valiosa para el profesor, pues le permite incorporar mejoras en el proceso de aprendizaje.

La dimensión Control, también evidencia logros, alcanzando un 25\% de mejora sobre la primera presentación. Manifestándose en actitudes más críticas sobre lo que 
el estudiante hs aprendido y aún le falta. Reconociendo lo que más le gusta hacer y en donde tiene mayor dificultades.

Los impactos del portafolio del estudiante en los buenos aprendizajes alcanzados por el grupo experimental, también se manifiestan en el aprendizaje actitudinal. Los resultados evidencian el paso de un nivel regular de aprendizaje a un nivel de bueno de aprendizaje (ver tabla 3).

Tabla 3 - Clasificación de los niveles de aprendizaje actitudinal a través de la Rúbrica

\begin{tabular}{|c|c|c|c|c|}
\hline Aprendizaje actitudinal & $\begin{array}{c}\text { Instrumento } \\
\text { Evaluativo }\end{array}$ & & Niveles & Calificación \\
\hline \multirow{4}{*}{$\begin{array}{l}\text { Denominado también saber } \\
\text { ser. Para la presente } \\
\text { investigación corresponde la } \\
\text { demostración o manifestación } \\
\text { de actitudes o valores } \\
\text { relacionados con la } \\
\text { Autonomía para decidir. } \\
\text { Habilidades sociales para } \\
\text { promover la solidaridad, el } \\
\text { compañerismo, el respeto y } \\
\text { valoración de la opinión de } \\
\text { otros. Responsabilidad, } \\
\text { cumplimiento y compromiso, } \\
\text { así como la comunicación y } \\
\text { defensa de sus ideas ante } \\
\text { terceros }\end{array}$} & \multirow{4}{*}{ Rúbrica } & Muy bueno & $\begin{array}{l}\text { Manifiesta todos los } \\
\text { indicadores actitudinales } \\
\text { establecidos }\end{array}$ & 4 \\
\hline & & Bueno & $\begin{array}{l}\text { Manifiesta dos } \\
\text { indicadores actitudinales } \\
\text { establecidos }\end{array}$ & 3 \\
\hline & & Regular & $\begin{array}{l}\text { Demuestra al menos un } \\
\text { indicador actitudinal } \\
\text { establecidos. }\end{array}$ & 2 \\
\hline & & Deficiente & $\begin{array}{l}\text { No demuestra ningún } \\
\text { indicador actitudinal } \\
\text { establecidos. }\end{array}$ & 1 \\
\hline
\end{tabular}

En el cuadro 4, se destacan la mejora porcentual del $31.2 \%$ en los promedios obtenidos sobre la primera presentación, superando el regular nivel de aprendizaje obtenido. 
Cuadro 4 - Promedios de los aprendizajes de contenidos actitudinales en la 1ra y 2da Presentación de los Portafolios

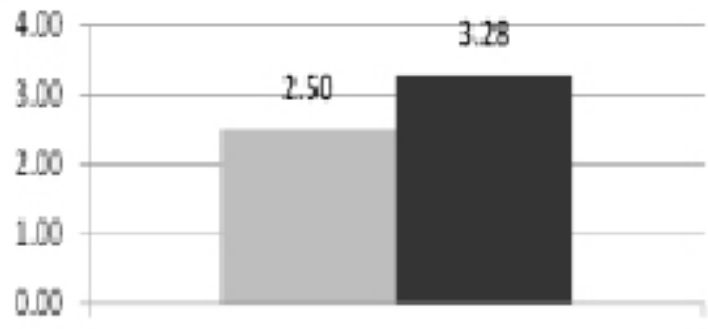

Promedios

IIragresentaoin I2dapresentacion

Los resultados porcentuales en los promedios de los tres indicadores que comprenden el aprendizaje actitudinal evidencian mejoras. El indicador Habilidades sociales pasa de un promedio 2.17 al promedio de 3,28 , esto es un 51,95\% sobre la primera presentación. El indicador Responsabilidad pasa de un promedio de 2.56 a un promedio de 3.89, es decir un 51,15\% mayor. El indicador Autonomía, sube de 2. 61 a 4, este logro de $53.25 \%$ permite alcanzar el máximo nivel (Ver cuadro 5).

Cuadro 5 - Promedios de los indicadores actitudinales en la 1ra y 2da presentación

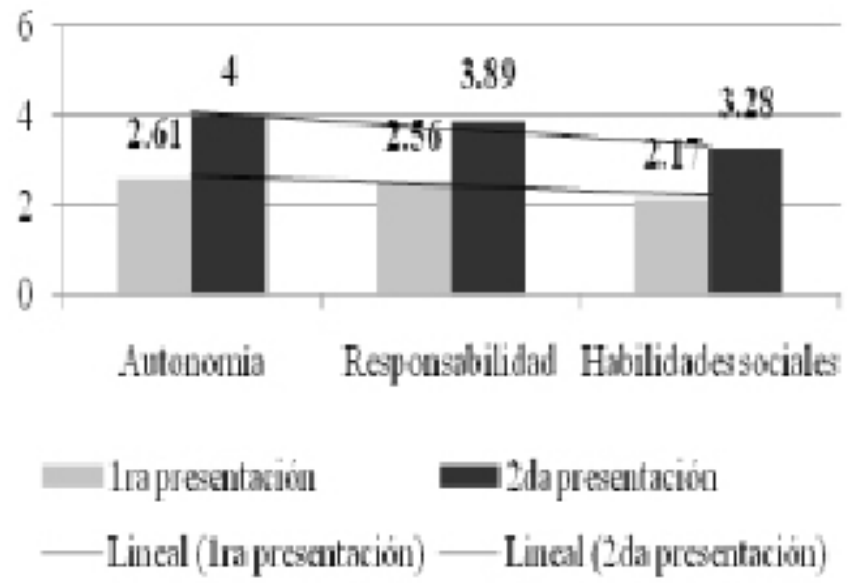

Todas estas características señaladas en los resultados obtenidos en la segunda presentación de los portafolios, evidencia en los estudiantes una mayor confianza y autonomía para tomar decisiones, elegir por si mismos sus evidencias, conciliar sus plazos y emplear criterios para la evaluación de sus trabajos. Se produce una mayor solidaridad entre ellos: se brindan más apoyo y cooperación. Sus portafolios contienen el aporte y contribución de otros compañeros en su aprendizaje. Sus habilidades investigativas evidencian la exploración de nuevas fuentes de información hechas por inicia- 
tiva propia. Los comentarios escritos señalan que se divierten aprendiendo. En general los estudiantes entregaron sus portafolios concluidos en la fecha acordada.

\section{CONSIDERACIONES FINALES}

El uso de los portafolios del estudiante en la disciplina de administración, evidencia mejoras significativas en el aprendizaje de contendidos, promoviendo el paso de un nivel regular de aprendizaje a un nivel bueno de aprendizaje de contenidos procedimentales, actitudinales y conceptuales, además del desarrollo de habilidades, reflejadas en una actitud autónoma por el estudio y la investigación.

Los aprendizajes de contenidos conceptuales logrados por el Grupo experimental, al aplicarse la prueba objetiva de 20 ítems del post-test, evidencia que los estudiantes obtienen un promedio final de 15,9 superando el promedio inicial de 6.8 y de 12,6 alcanzado por el Grupo control.

Los estudiantes evidencian la adquisición de habilidades y capacidades para planificar, organizar, dirigir y controlar el uso de las evidencias de sus portafolios en el conocimiento y aplicación de los conceptos, técnicas y herramientas de la administración en la resolución de problemas relacionados con la toma de decisiones y mejor organización del trabajo y manejo del tiempo. A utilizarlo aprendieron a plantear sus propias metas, planes y actividades para alcanzar dichos objetivos, jerarquizando sus prioridades, contando con una mayor disposición para reflexionar, trabajar cooperativamente, valorar y respetar opiniones diferentes. Teniendo una actitud más responsable sobre la tarea de aprender de manera autónoma y regulada.

Los estudiantes reconocen el impacto positivo del uso de los portafolios en sus vidas, configurando una nueva identidad, reflejando una mayor responsabilidad sobre su propio aprendizaje. Descubriendo satisfacción por aprender e investigar, transformando sus debilidades en oportunidades para ser más creativos y disfrutar descubriendo nuevas formas de superarse y crecer como personas. Afirman que al construir sus portafolios se genero un dialogo más abierto entre ellos y con el profesor. Mejorando el uso adecuado de los recursos bibliográficos y audiovisuales. Registrando sus ocurrencias y la capacidad para autoevaluarse y coevaluar a otros.

El impacto del uso de los portafolios del estudiante en la disciplina de administración se manifiesta en una notoria mejoría en sus aprendizajes actitudinales y procedimentales principalmente. 


\section{REFERENCIAS}

ALTAMIRANO CÁRDENAS, C. Estrategias de aprendizaje y el Rendimiento académico en estudiantes de la escuela académico profesional de medicina humana en la Universidad Peruana los Andes, Huancayo. Lima, Perú. 2006. 160 Pág. Tesis (Maestría en Docencia en Educación Superior) - Facultad de Educación, Universidad Peruana Cayetano Heredia.

BARTON, J. e COLLINS, A. Portfolio assessment: a handbook for educators. Nova York: Dale Seymour Publications. Usa. 1997.

BENITO A, CRUZ A y otros. Nuevas claves para la docencia universitaria en el espacio europeo de educación superior. Narcea Ediciones. 2005.

CASTRO, L. El portafolio de enseñanza como herramienta y texto para la reflexión pedagógica, en Perspectiva Educativa, nº 3., Universidad del Tolima: Ibagué. 2002.

DANIELSON, Charlotte y ABRUTYN, Leslye. Una introducción al uso de portafolio en el aula, México: Fondo de Cultura Económica. 2004.

GARCÍA, E. Algunas aplicaciones del portafolio en el ámbito educativo. Secretaría de Educación y Cultura del Estado de Chihuahua. México. 2000.

GATICA, F., OREA, F. R. y VEGA, M. F. (2007). E-portafolio como recurso académico en Medicina. Revista digital universitaria, 4. 2007. Disponible en <http:/ / www.revista.unam.mx/ forodigital3programa.htm> acceso 21 marzo de 2012.

HERNÁNDEZ, F. Cultura visual, mudança educativa e projeto de trabalho. Porto Alegre: Artes Médicas Sul. 2000.

HILDA, E. Q. El portafolio como estrategia para la evaluación. Teoría y didáctica de la lengua y de la literatura. Universidad Interamericana de Puerto Rico, abril 1996. $\mathrm{n}^{\circ}$ 8, pp 89- 96.

KLENOWSKI, V. Desarrollo del portafolio para el aprendizaje y la evaluación. Madrid: Narcea. 2003.

LYONS, N. (comp.). El uso de portafolios. Propuestas para un nuevo profesionalismo docente, Buenos Aires: Amorrortu Nunan, D. (1999): "Case Study", en Research Methods in Language.

MELLADO, M. Grado de satisfacción en relación con el portafolio en línea como herramienta para evaluar la formación inicial docente. Boletín de investigación Educacional. 2005. n20 (2), pp. 231-250 Santiago: Facultad de Educación. Pontificia Universidad Católica de Chile.

PINAR SEPÚLVEDA, Ma ÁNGELES Y GRACIA MORÁN JOAQUÍN. Aplicación del Portafolio como estrategia de evaluación formativa. Departamento de Informática de Sistemas y Computadores (DISCA) Escuela Técnica Superior de Informática Aplicada (ETSIA). Universidad Politécnica de Valencia (UPV). 2006. Disponible en <http://jenui2007.unizar.es/doc/Sesiones/17-2B/JGracia_Portafolio_alua2.1.pdf> acceso 18 marzo de 2012.

REZENDE, R. Márcia A. (2010). La relación pedagógica y evaluación en El espejo Del portafolio: memórias docente y discente. Minas Gerais, 2010. Tesis (Doctorado en Educación) - Facultad de Educación, Universidad Federal de Minas Gerais.

SÁ-CHAVES, I. Portafolios reflexivos: estrategia de formación y supervisión. Aveiro:

Universidad de Aveiro. Cuadernos didácticos. Serie Superior. 2000. 
SELDIN, P. The teaching portfolio: a practical guide to improve performance and promotion/tenure decisions, Bolton: Anker Publishing Company. 2004.

VILLAS BOAS, M. F. Evaluación formativa: en busca del desenvolvimiento del alumno, del profesor y la escuela. In: VEIGA, Ilma P.A. e FONSECA, Marília (orgs.). Las dimensiones Del proyecto político - pedagógico: Nuevos desafíos para la escuela. Campinas: Papirus. 2001.

Portafolio, evaluación y trabajo pedagógico. 3ra edición. 2006.

VILLALUSTRE, Lourdes y $\mathrm{M}^{\mathrm{a}}$ DEL MORAL PÉREZ MARTINEZ, Esther. Eportafolios y rúbricas de evaluación en ruralnet. Revista de Medios y Educación. ISSS: 1133-8482 - $\mathrm{N}^{\mathrm{o}} 37$ Julio - Diciembre 2010. pp. 93 - 105. Disponible en < http://www.sav.us.es/pixelbit/pixelbit/articulos/n37/8.pdf> Acceso el 17 marzo de 2012. 